\author{
M.G. Mazhgikhova \\ Institute of Applied Mathematics and Automation, Nalchik, Russia \\ (E-mail: mazhgihova.madina@yandex.ru)
}

\title{
Green function method for a fractional-order delay differential equation
}

\begin{abstract}
In this paper, we investigated a boundary value problem with the Sturm-Liouville type conditions for a linear ordinary differential equation of fractional order with delay. The condition for the unique solvability of the problem is obtained in the form $\triangle \neq 0$. The Green function of the problem, in terms of which the solution of the boundary value problem under study is written out, is constructed. The existence and uniqueness theorem for the solution of the problem is proved. It is also showed that in the case when the condition of unique solvability is violated, i.e $\Delta=0$, then the solution of the boundary value problem is not unique. Using the notation of the generalized Mittag-Leffler function via the generalized Wright function, we also studied the properties of the function $\triangle$ as $\lambda \rightarrow \infty$ and $\lambda \rightarrow-\infty$. Using asymptotic formulas for the generalized Wright function, a theorem on the finiteness of the number of eigenvalues of a boundary value problem with the Sturm-Liouville type conditions is proved.
\end{abstract}

Keywords: Fractional differential equation, delay differential equation, Green function, generalized MittagLeffler function, generalized Wright function.

\section{Introduction}

Consider the equation

$$
\frac{d^{\alpha}}{d t^{\alpha}} u(t)-\lambda u(t)-\mu H(t-\tau) u(t-\tau)=f(t), \quad 0<t<1
$$

where $\frac{d^{\alpha}}{d t^{\alpha}}$ is the Riemann-Liouville fractional derivative [1], $1<\alpha \leq 2, \lambda, \mu$ are the arbitrary constants, $\tau$ is the fixed positive number, $H(t)$ denotes the Heaviside function.

At present, the number of studies on fractional calculation has noticeably increased. This is due to the fact that fractional order differential equations are used in mathematical modeling of processes that occur in various fields of natural science, such as physics, chemistry, biology, sociology, etc.

The most general references to the theory of fractional calculus one can find in [2-5] (see also the references in these works). A linear ordinary differential equation of fractional order was considered by Barrett [6] in 1954 . Existence and uniqueness theorem for a fractional-order differential equation is proved in [7] by Dzhrbashyan and Nersesyan. Sturm-Liuville type boundary value problem for fractional differential operator was investigated by Dzhrbashyan in [8]. The initial value problem for a linear ordinary differential equation of fractional order was studied by Pskhu in [9].

Significant works were devoted to the delay differential equations (difference-differential equations) by Norkin in [10], Bellman and Cooke in [11], Elsgolts and Norkin in [12], Myshkis [13], Hale in 1977 [14].

The initial-value problem and the problem with general linear two-point boundary conditions, the Dirichlet and the Neumann problems for linear ordinary differential equation with Caputo derivative with delay in [15-17] respectevely were solved.

The Cauchy problem for Eq.(1) was considered in [18], and the solutions to the Dirichlet and the Neumann problems were obtained in [19].

In this paper, we construct the Green function of the Sturm-Liouville type boundary value problem for Eq.(1) and prove the finiteness theorem for the number of real eigenvalues of the study problem. 


\section{Auxiliary}

The Riemann-Liouville fractional operator is define by the formula

$$
\frac{d^{\alpha}}{d t^{\alpha}} u(t)=D_{a t}^{\alpha} u(t)=\frac{1}{\Gamma(n-\alpha)} \frac{d^{n}}{d t^{n}} \int_{a}^{t} \frac{u(\xi) d \xi}{(t-\xi)^{\alpha-n+1}}, \quad n-1<\alpha \leq n, n \in \mathbb{N},
$$

where $\Gamma(z)=\int_{0}^{\infty} e^{-t} t^{z-1} d t$ is the Euler gamma function.

The Mittag-Leffler function is given by the power series [20]

$$
E_{\alpha, \beta}(z)=\sum_{k=o}^{\infty} \frac{z^{k}}{\Gamma(\alpha k+\beta)}
$$

and the generalized Mittag-Leffler function defines by the series [21]

$$
E_{\alpha, \beta}^{\rho}=\sum_{k=o}^{\infty} \frac{(\rho)_{k} z^{k}}{\Gamma(\alpha k+\beta) k !}
$$

where $(\rho)_{k}=\frac{\Gamma(\rho+k)}{\Gamma(\rho)}$ is the Pokhhammer symbol. The generalized Mittag-Leffler function reduces to $E_{\alpha, \beta}(z)$ when we set $\rho=1$.

Consider the function

$$
W_{\nu}(t)=W_{\nu}(t, \tau ; \lambda, \mu)=\sum_{m=0}^{\infty} \mu^{m}(t-m \tau)_{+}^{\alpha m+\nu-1} E_{\alpha, \alpha m+\nu}^{m+1}\left(\lambda(t-m \tau)_{+}^{\alpha}\right), \nu \in \mathbb{R}
$$

where

$$
(t-m \tau)_{+}=\left\{\begin{aligned}
t-m \tau, & t-m \tau>0 \\
0, & t-m \tau \leq 0 .
\end{aligned}\right.
$$

It follows from (2) that

$$
W_{k}^{(i)}(0)=\left\{\begin{array}{l}
0, k \neq i+1, \\
1, k=i+1 .
\end{array}\right.
$$

Remark 1. For some $m$ the expression $t-m \tau<0$, therefore the series in (2) contains a finite number of terms $N \leq\left[\frac{t}{\tau}\right]+1$.

Function (2) satisfies the following properties [16]

$$
\begin{gathered}
D_{0 t}^{\alpha} W_{\nu}(t)=W_{\nu-\alpha}(t), \quad \alpha \in \mathbb{R}, \quad \nu>0, \\
W_{\nu}(t)=\lambda W_{\nu+\alpha}(t)+\mu W_{\nu+\alpha}(t-\tau)+\frac{t^{\nu-1}}{\Gamma(\nu)}, \quad \alpha>0, \quad \nu \in \mathbb{R},
\end{gathered}
$$

which are clear by the formula of differentiation [21]

$$
\left.\frac{d^{m}}{d z^{m}}\left(z^{\beta-1} E_{\alpha, \beta}^{\rho}\left(z^{\alpha}\right)\right)=z^{\beta-m-1} E_{\alpha, \beta-m}^{\rho}\left(z^{\alpha}\right)\right)
$$

and by the autotransformation formula [22]:

$$
E_{\alpha, \beta}^{\rho}(t)-E_{\alpha, \beta}^{\rho-1}(t)=t E_{\alpha, \alpha+\beta}^{\rho}(t)
$$

of the generalized Mittag-Leffler function. 


\section{Main results}

A function $u(t)$ is called a regular solution of Eq.(1) if $D_{0 t}^{\alpha-2} u(t) \in C^{2}(0,1), u(t) \in L(0,1)$ and $u(t)$ satisfies Eq. (1) for all $0<t<1$.

The problem we solve here is to find the regular solution to equation (1) satisfying the conditions

$$
\begin{aligned}
& a \lim _{t \rightarrow 0} D_{0 t}^{\alpha-1} u(t)+b \lim _{t \rightarrow 0} D_{0 t}^{\alpha-2} u(t)=0, \\
& c \lim _{t \rightarrow 1} D_{0 t}^{\alpha-1} u(t)+d \lim _{t \rightarrow 1} D_{0 t}^{\alpha-2} u(t)=0,
\end{aligned}
$$

where $a^{2}+b^{2} \neq 0$ and $c^{2}+d^{2} \neq 0$.

\section{Green function}

Assume $G(t, \xi)$ is given by

$$
G(t, \xi)=H(t-\xi) W_{\alpha}(t-\xi)+\left(c W_{1}(1-\xi)+d W_{2}(1-\xi)\right) \frac{b W_{\alpha}(t)-a W_{\alpha-1}(t)}{\triangle}
$$

with $\lambda$ and $\mu$ satisfying the following condition

$$
\triangle=a c\left(\lambda W_{\alpha}(1)+\mu W_{\alpha}(1-\tau)\right)+(a d-b c) W_{1}(1)-b d W_{2}(1) \neq 0 .
$$

Here the function $W_{\nu}(t)$ is defined via $(2)$.

We demonstrate the validity of the following properties for the function $G(t, \xi)(7)$.

1. The function $G(t, \xi)$ is continuous for all values of $t$ and $\xi$ from the closed interval $[0,1]$.

This property implies from relation (7) and condition (8).

2. The function $G(t, \xi)$ satisfies the conditions

$$
\lim _{\varepsilon \rightarrow 0}\left[\left.D_{0 t}^{\alpha-2} G_{\xi}(t, \xi)\right|_{\xi=t+\varepsilon}-\left.D_{0 t}^{\alpha-2} G_{\xi}(t, \xi)\right|_{\xi=t-\varepsilon}\right]=1
$$

Indeed,

$$
\begin{gathered}
D_{0 t}^{\alpha-2} G_{\xi}(t, \xi)=-H(t-\xi) W_{1}(t-\xi) \\
-\left(c \lambda W_{\alpha}(1-\xi)+c \mu W_{\alpha}(1-\xi-\tau)+d W_{1}(1-\xi)\right) \frac{b W_{2}(t)-a W_{1}(t)}{\triangle} .
\end{gathered}
$$

Insert (10) into (9) as $\xi=t+\varepsilon$ and $\xi=t-\varepsilon$. Passing to the limit as $\varepsilon \rightarrow 0$ we get the property (9).

3. The function $G(t, \xi)$ is the solution to the equation

$$
\partial_{1 \xi}^{\alpha} G(t, \xi)-\lambda G(t, \xi)-\mu H(1-\tau-\xi) G(t, \xi+\tau)=0 .
$$

Here $\partial_{0 t}^{\alpha}$ is the Caputo derivative $[23 ; 11]$ defines as

$$
\partial_{0 t}^{\alpha} u(t)=D_{0 t}^{\alpha-2} u^{\prime \prime}(t)=\frac{1}{\Gamma(2-\alpha)} \int_{0}^{t} \frac{u^{\prime \prime}(\xi) d \xi}{(t-\xi)^{\alpha-1}} .
$$

This property implies the presentation of the function (7) and the relations (4), (5).

4. The function $G(t, \xi)$ satisfies the boundary conditions

$$
\left\{\begin{array}{l}
a \lim _{\xi \rightarrow 0} D_{0 t}^{\alpha-2} G_{\xi}(t, \xi)+b \lim _{\xi \rightarrow 0} D_{0 t}^{\alpha-2} G(t, \xi)=0 \\
c \lim _{\xi \rightarrow 1} D_{0 t}^{\alpha-2} G_{\xi}(t, \xi)+d \lim _{\xi \rightarrow 1} D_{0 t}^{\alpha-2} G(t, \xi)=0
\end{array}\right.
$$

This property obviously implies the relations (4), (5).

The function $G(t, \xi)$ that possesses properties 1-4 is called Green function for problem (1), (6). 


\section{Existence and uniqueness theorem}

Theorem 1. Assume the function $f(t) \in L(0,1) \cap C(0,1)$ and the condition (8) is satisfied. Then 1) there exists a regular solution to problem (1), (6) in the form of

$$
u(t)=\int_{0}^{1} f(\xi) G(t, \xi) d \xi
$$

2) the solution to problem (1), (6) is unique if and only if condition (8) is satisfied.

Proof. First we illustrate that the solution to problem (1), (6) has the form (13). To clear this, multiply both sides of Eq. (1) (given in terms of variable $\xi$ ) by $D_{0 t}^{\alpha-2} G(t, \xi)$ and integrate it with respect to variable $\xi$ ranging from $\varepsilon$ to $1-\varepsilon(\varepsilon \rightarrow 0)$ :

$$
\begin{gathered}
\int_{\varepsilon}^{1-\varepsilon} D_{0 t}^{\alpha-2} G(t, \xi) D_{0 \xi}^{\alpha} u(\xi) d \xi-\lambda \int_{\varepsilon}^{1-\varepsilon} u(\xi) D_{0 t}^{\alpha-2} G(t, \xi) d \xi \\
-\mu \int_{\varepsilon}^{1-\varepsilon} H(t-\tau) u(\xi-\tau) D_{0 t}^{\alpha-2} G(t, \xi) d \xi=\int_{\varepsilon}^{1-\varepsilon} f(\xi) D_{0 t}^{\alpha-2} G(t, \xi) d \xi, \quad 0<t<1 .
\end{gathered}
$$

Integrate by parts the first term of equality (14):

$$
\begin{gathered}
\int_{\varepsilon}^{1-\varepsilon} D_{0 t}^{\alpha-2} G(t, \xi) D_{0 \xi}^{\alpha} u(\xi) d \xi=\left.D_{0 t}^{\alpha-2} G(t, \xi) D_{0 \xi}^{\alpha-1} u(\xi)\right|_{\varepsilon} ^{1-\varepsilon}-\int_{\varepsilon}^{t} D_{0 t}^{\alpha-2} G_{\xi}(t, \xi) D_{0 \xi}^{\alpha-1} u(\xi) d \xi \\
-\int_{t}^{1-\varepsilon} D_{0 t}^{\alpha-2} G_{\xi}(t, \xi) D_{0 \xi}^{\alpha-1} u(\xi) d \xi=\left.D_{0 t}^{\alpha-2} G(t, \xi) D_{0 \xi}^{\alpha-1} u(\xi)\right|_{\xi=1}-\left.D_{0 t}^{\alpha-2} G(t \xi) D_{0 \xi}^{\alpha-1} u(\xi)\right|_{\xi=0} \\
+D_{0 t}^{\alpha-2} u(\xi)\left[\left.D_{0 t}^{\alpha-2} G_{\xi}(t, \xi)\right|_{\xi=t+0}-\left.D_{0 t}^{\alpha-2} G_{\xi}(t, \xi)\right|_{\xi=t-0}\right]+\left.D_{0 t}^{\alpha-2} G_{\xi}(t, \xi) D_{0 \xi}^{\alpha-2} u(\xi)\right|_{\xi=0} \\
-\left.D_{0 t}^{\alpha-2} G_{\xi}(t, \xi) D_{0 \xi}^{\alpha-2} u(\xi)\right|_{\xi=1}+\int_{0}^{1} D_{0 t}^{\alpha-2} G_{\xi \xi}(t, \xi) D_{0 \xi}^{\alpha-2} u(\xi) d \xi .
\end{gathered}
$$

Applying to (15) the properties (9), (12) of function (7) and conditions (6) of the problem we get the following formula

$$
D_{0 t}^{\alpha-2} u(\xi)+\int_{0}^{1} D_{0 \xi}^{\alpha-2} u(\xi) D_{0 t}^{\alpha-2} G_{\xi \xi}(t, \xi) d \xi .
$$

Replace $\xi$ with $\xi-\tau$ in the third integral on the left-hand side of the expression (14) to reduce it to

$$
\int_{0}^{1} H(\xi-\tau) u(\xi-\tau) G(t, \xi) d \xi=\int_{0}^{1} H(1-\tau-\xi) u(\xi) G(t, \xi+\tau) d \xi .
$$

Put (16) and (17) into Eq. (14) and using the formula for fractional integration by parts [20, p. 15]

$$
\int_{a}^{b} g(s) D_{a s}^{\alpha} h(s) d s=\int_{a}^{b} h(s) D_{b s}^{\alpha} g(s) d s
$$

arrive at identity

$$
D_{0 t}^{\alpha-2} u(\xi)+D_{0 t}^{\alpha-2} \int_{0}^{1} u(\xi)\left[D_{1 \xi}^{\alpha-2} G_{\xi \xi}(t, \xi)-\lambda G(t, \xi)-\mu H(1-t-\xi) G(t, \xi+\tau)\right] d \xi=
$$




$$
=D_{0 t}^{\alpha-2} \int_{0}^{1} f(\xi) G(t, \xi) d \xi
$$

Taking advantage of the third property of Green function $G(t, \xi)(11)$ and finding the derivative of order $D_{0 t}^{2-\alpha}$ we arrive at representation (13).

Next, we show that the function (13) is the solution to problem (1), (6). Formula (13) can be written out in terms of function $W_{\nu}(t)$ in the form of bellow:

$$
u(t)=\int_{0}^{t} f(\xi) W_{\alpha}(t-\xi) d \xi+\frac{b W_{\alpha}(t)-a W_{\alpha-1}(t)}{\triangle} \int_{0}^{1} f(\xi)\left(c W_{1}(1-\xi)+d W_{2}(1-\xi)\right) d \xi .
$$

Next, using formulas (4), (5) obtain by the previous relation that

$$
D_{0 t}^{\alpha} u(t)=f(t)+\lambda \int_{0}^{1} f(\xi) G(t, \xi) d \xi+\mu \int_{0}^{1} f(\xi) G(t, \xi-\tau) d \xi .
$$

Prove that the solution $u(t)$ satisfies the boundary conditions (6) (in view of relation (3)):

$$
\begin{gathered}
a \lim _{t \rightarrow 0} D_{0 t}^{\alpha-1} u(t)+b \lim _{t \rightarrow 0} D_{0 t}^{\alpha-2} u(t)=\frac{1}{\triangle} \int_{0}^{1} f(\xi)\left[c W_{1}(1-\xi)+d W_{2}(1-\xi)\right] \\
\times\left[a b W_{1}(0)-a^{2} \lambda W_{\alpha}(0)-a^{2} \mu W_{\alpha}(-\tau)+b^{2} W_{2}(0)-a b W_{1}(0)\right] d \xi=0 \\
c \lim _{t \rightarrow 1} D_{0 t}^{\alpha-1} u(t)+d \lim _{t \rightarrow 1} D_{0 t}^{\alpha-2} u(t)=\int_{0}^{1} f(\xi)\left[c W_{1}(1-\xi)+d W_{2}(1-\xi)\right] \times \\
\times\left[1+\frac{-a c\left(\lambda W_{\alpha}(1)+\mu W_{\alpha}(1-\tau)\right)-(a d-c b) W_{1}(1)+b d W_{2}(1)}{\triangle}\right] d \xi= \\
=\int_{0}^{1} f(\xi)\left(c W_{1}(1-\xi)+d W_{2}(1-\xi)\right)\left(1-\frac{\triangle}{\triangle}\right) d \xi=0 .
\end{gathered}
$$

The task is now to show that if the condition (8) is not satisfied

$$
\triangle=0,
$$

then the solution of the problem is not unique. Consider the function

$$
\bar{u}(t)=C_{1} W_{\alpha}(t)+C_{2} W_{\alpha-1}(t),
$$

which is the solution to the problem

$$
\begin{aligned}
& D_{0 t}^{\alpha} \bar{u}(t)-\lambda \bar{u}(t)-\mu H(t-\tau) \bar{u}(t-\tau)=0, \\
& a \lim _{t \rightarrow 0} D_{0 t}^{\alpha-1} \bar{u}(t)+b \lim _{t \rightarrow 0} D_{0 t}^{\alpha-2} \bar{u}(t)=0, \\
& c \lim _{t \rightarrow 1} D_{0 t}^{\alpha-1} \bar{u}(t)+d \lim _{t \rightarrow 1} D_{0 t}^{\alpha-2} \bar{u}(t)=0 .
\end{aligned}
$$

The conditions (18) can be written out in the form

$$
\begin{gathered}
a C_{1}+b C_{2}=0 \\
C_{1}\left[W_{1}(1)+d W_{2}(1)\right]+C_{2}\left[c \lambda W_{\alpha}(1)+c \mu W_{\alpha}(1-\tau)+d W_{1}(1)\right]=0 .
\end{gathered}
$$

Then the determinant of the system (19) is equal to

$$
\bar{\triangle}=\left|\begin{array}{cc}
a & b \\
c W_{1}(1)+d W_{2}(1) & c \lambda W_{\alpha}(1)+c \mu W_{\alpha}(1-\tau)+d W_{1}(1)
\end{array}\right|=0 .
$$


Thus, solution to problem (1), (6) is unique if and only if condition (8) is satisfied.

Remark. For all

$$
\lambda, \mu>0, \quad(a-b)(c+d)>0
$$

condition (8) is always satisfied.

\section{On the finiteness of the number of real eigenvalues}

Definition. The eigenvalues of problem (1), (6) are the values $\lambda$, such that problem (1), (6) has a regular solution that is not the identically zero.

The set of real eigenvalues for problem (1), (6) coincides with the set of real zeros for the function

$$
\Phi(\lambda)=a c\left(\lambda W_{\alpha}(1)+\mu W_{\alpha}(1-\tau)\right)+(a d-b c) W_{1}(1)-b d W_{2}(1) .
$$

Theorem 2. Problem (1), (6) has only a finite number of real eigenvalues.

The function $W_{\nu}(\lambda)$ can be written out as $[2$, p. 45$]$

$$
W_{\nu}(1, \tau ; \lambda, \mu)=\sum_{m=0}^{\infty} \frac{\mu^{m}}{m !}(1-m \tau)_{+}^{\alpha m+\nu-1}{ }_{1} \Psi_{1}\left[\begin{array}{c}
(m+1,1) \\
(\alpha m+\nu, \alpha)
\end{array} \mid \lambda(1-m \tau)_{+}^{\alpha}\right],
$$

where

$$
{ }_{p} \Psi_{q}\left[\begin{array}{c}
\left(a_{l}, \alpha_{l}\right)_{1, p} \\
\left(b_{l}, \beta_{l}\right)_{1, q}
\end{array} \mid z\right]=\sum_{k=0}^{\infty} \frac{\prod_{l=1}^{p} \Gamma\left(a_{l}+\alpha_{l} k\right)}{\prod_{l=1}^{q} \Gamma\left(b_{l}+\beta_{l} k\right)} \frac{z^{k}}{k !}
$$

is the generalized Wright function [24].

Function (20) is an integer function of parameter $\lambda$. Let us investigate the properties of the function (20) as $\lambda \rightarrow+\infty$ and $\lambda \rightarrow-\infty$.

As $\lambda \rightarrow+\infty$ the following asymptotic formula holds true for the generalized Wright function [24], [25]:

$$
{ }_{1} \Psi_{1}\left[\begin{array}{c}
(m+1,1) \\
(\alpha m+\nu, \alpha)
\end{array} \mid \lambda(1-m \tau)_{+}^{\alpha}\right]=\alpha^{-m} \lambda^{\frac{m(1-\alpha)-\nu+1}{\alpha}}(1-m \tau)_{+}^{m(1-\alpha)-\nu+1} e^{\lambda^{1 / \alpha}(1-m \tau)_{+}}\left[1+O\left(\frac{1}{\lambda^{\frac{1}{\alpha}}}\right)\right] .
$$

Let $N$ be the maximum value of $m$ that satisfies the inequality $(1-m \tau)>0$. Then the asymptotic formula for function (20) is in the form

$$
\begin{gathered}
\Phi(\lambda)=\sum_{m=0}^{\infty} \frac{\mu^{m} \alpha^{-m}}{m} \lambda^{\frac{m-\alpha m+1}{\alpha}}\left[(1-m \tau)_{+}^{m} e^{\lambda^{1 / \alpha}(1-m \tau)+}\left(a c+(a d-b c) \lambda^{-1 / \alpha}-b d \lambda^{-2 / \alpha}\right)\right. \\
\left.+a c \frac{\mu}{\lambda}(1-(m+1) \tau)_{+}^{m} e^{\lambda^{1 / \alpha}(1-(m+1) \tau)}\right] \times\left[1+O\left(\lambda^{-1 / \alpha}\right)\right] .
\end{gathered}
$$

Hence, as $\lambda \rightarrow \infty$, the series above increases without limit.

The asymptotic formula for the generalized Wright function as $\lambda \rightarrow-\infty$ has form [24], [25]

$$
\begin{aligned}
{ }_{1} \Psi_{1}\left[\begin{array}{c}
(m+1,1) \\
(\alpha m+\nu, \alpha)
\end{array} \mid \lambda(1-m \tau)_{+}^{\alpha}\right] & =\sum_{l=0}^{n} \frac{(-1)^{m+l+1}(l+m) !(1-m \tau)_{+}^{-\alpha(m+l+1)}}{|\lambda|^{m+l+1} \Gamma(\nu-\alpha-\alpha l)(m+l+1) !} \\
& +O\left(\frac{1}{|\lambda|^{m}}\right) .
\end{aligned}
$$

Therefore

$$
\begin{aligned}
\Phi(\lambda)= & \sum_{m=0}^{N} \frac{(-1)^{m+1} \mu^{m}}{(m+1) !|\lambda|^{m}}\left[\frac{a c}{\Gamma(-\alpha)}\left((1-m \tau)_{+}^{-1}+\frac{\mu\left(1-(m+1)_{+} \tau\right)^{-1}}{|\lambda|}\right)\right. \\
& \left.+(a d-b c) \frac{(1-m \tau)^{-\alpha}}{|\lambda| \Gamma(1-\alpha)}-b d \frac{(1-m \tau)^{1-\alpha}}{|\lambda| \Gamma(2-\alpha)}+O\left(\frac{1}{|\lambda|^{N+1}}\right)\right] .
\end{aligned}
$$

Consider the limit relation in the case when $\mu \neq 0$

$$
\lim _{\lambda \rightarrow-\infty} \lambda^{N} \Phi(\lambda)=\frac{a c(-1)^{N+1} \mu^{N}(1-N \tau)_{+}^{-1}}{\Gamma(-\alpha)(N+1) !} \neq 0 .
$$


As $\mu=0$ we have

$$
\lim _{\lambda \rightarrow-\infty} \lambda \Phi(\lambda)=-\frac{a c}{\Gamma(-\alpha)}
$$

Since $\Phi(\lambda)$ is an entire function of the variable $\lambda$, it follows from relations (21), (22), and (23) that the series (20) may have only a finite number of real zeros. This establishes the theorem.

\section{References}

1 Podlubny I. Fractional differential equations / I. Podlubny. - AP, New York, 1999. - 341 p.

2 Kilbas A.A. Theory and Applications of Factional Differential Equations / A.A. Kilbas, H.M. Srivastava, J.J. Trujillo. - Elsevier, Amsterdam, 2006. - 523 p.

3 Псху А.В. Уравнения в частных производных дробного порядка / А.В. Псху. - М.: Наука, 2005. $199 \mathrm{c.}$

4 Oldham K.B. The fractional calculus / K.B. Oldham, J. Spanier. - N.-Y. L.: AP, 1974. - 234 p.

5 Miller K.S. An introduction to the fractional calculus and fractional differential equations / K.S. Miller, B. Ross. - John Wiley \& sons, New York, 1993. - 366 p.

6 Barrett J.H. Differential equation of non-integer order / J.H. Barrett // Canad. J. Math. - 1954. - Vol. 6, No. 4. - P. 529-541.

7 Джрбашян М.М. Дробные производные и задача Коши для дифференциальных уравнений дробного порядка / М.М. Джрбашян, А.Б. Нерсесян // Известия Академии наук Армянской ССР. - 1968. Т. 3, № 1. - C. 3-28.

8 Джрбашян М.М. Краевая задача для дифференциального оператора дробного порядка типа Штурма-Лиувилля / М.М. Джрбашян // Известия Академии наук Армянской ССР. - 1970. - T. 5, № 2. - C. 71-96.

9 Pskhu A.V. Initial-value problem for a linear ordinary differential equation of noninteger order / A.V. Pskhu // Sbornik: Mathematics. - 2011. - Vol. 202, No. 4. - P. 571-582.

10 Норкин С.Б. О решениях линейного однородного дифференциального уравнения второго порядка с запаздывающим аргументом / С.Б. Норкин // Успехи математических наук. - 1959. - Т. 14 , № 1. - C. 199-206.

11 Bellman R.E. Differential-Difference Equations / R.E. Bellman, K.L. Cooke. - Acad. Press, New YorkLondon, 1963. - $462 \mathrm{p}$.

12 Эльсгольц Л.Э. Введение в теорию дифференциальных уравнений с запаздывающим аргументом / Л.Э. Эльсгольц, С.Б. Норкин. - М.: Наука, 1971. - 296 с.

13 Мышкис А.Д. Линейные дифференциальные уравнения с запаздывающим аргументом / А.Д. Мышкис. - М.: Наука, 1972. - 352 с.

14 Hale J.K. Introduction to Functional Differential Equations / J.K. Hale, S.M.V. Lunel. - Springer, New-York, 1993. - 448 p.

15 Мажгихова М.Г. Начальная и краевая задачи для обыкновенного дифференциального уравнения дробного порядка с запаздывающим аргументом / М.Г. Мажгихова // Челяб. физ.-матем. журн. 2018. - T. 3, № 1. - C. 27-37.

16 Mazhgikhova M.G. Dirichlet Problem for a Fractional-Order Ordinary Differential Equation with Retarded Argument / M.G. Mazhgikhova // Differential Equations. - 2018. - Vol. 54, No. 2. - P. $185-192$.

17 Мажгихова М.Г. Задача Неймана для обыкновенного дифференциального уравнения дробного порядка с запаздывающим аргументом / М.Г. Мажгихова // Известия Кабардино-Балкарского научного центра РАН. - 2016. - Т. 2. - № 70. - С. 15-20.

18 Мажгихова М.Г. Задача Коши для обыкновенного дифференциального уравнения с оператором Римана-Лиувилля с запаздывающим аргументом / М.Г. Мажгихова // Известия КабардиноБалкарского научного центра РАН. - 2017. - Т. 1, № 75. - С. 24-28.

19 Мажгихова М.Г. Краевые задачи для линейного обыкновенного дифференциального уравнения дробного ряда с запаздывающим аргументом / М.Г. Мажгихова // Сибир. электрон. матем. изв. - 2018. - T. 15. - C. $685-695$. 
20 Wiman A. Über den Fundamentalsatz in der Teorie der Funktionen $E_{\alpha}(x) /$ A. Wiman // Acta Mathematica. - 1905. - Vol. 29, No. 1. - P. 191-201.

21 Prabhakar T.R. A singular integral equation with a generalized Mittag-Leffler function in the kernel / T.R. Prabhakar // Yokohama Math. J. - 1971. - Vol. 19. - P. 7-15.

22 Shukla A.K., Prajapati J.C. On a generalization of Mittag-Leffler function and its properties A.K. Shukla, J.C. Prajapati // J. Math. Anal. Appl. - 2007. - Vol. 336. - P. 797-811.

23 Нахушев А.М. Дробное исчисление и его применение / А.М. Нахушев. - М.: Физматлит, 2003. $272 \mathrm{c}$.

24 The asymptotic expansion of the generalized hypergeometric function / E.M. Wright // J. London Math. Soc. - 1935. - Vol. 10. - P. 286-293.

25 Wright E.M. The asymptotic expansion of the generalized hypergeometric function / E.M. Wright // Proc. London Math. Soc. - 1940. - Vol. 46, No. 2. - P. 389-408.

\title{
М.Г. Мажгихова
}

\section{Бөлшек ретті кешікпелі аргументті дифференциалдық теңдеу үшін Грин функциясы әдісі}

\begin{abstract}
Мақалада кәдімгі сызықтық тұрақты коэффициентті кешікпелі аргументті бөлшек ретті дифференциалдық теңдеу үшін Штурм-Лиувилль типті шеттік есеп зерттелген. Қойылған есептің бірмәнді шешілуі $\triangle \neq 0$ түрінде алынды. Зерттеліп отырған есепті шешу үшін Грин әдісі қолданылды. Грин функциялары Миттаг-Леффлер жалпыланған функциялары терминінде жазылды. Зерттеліп отырған есептің шешуінің бар болуы және жалғыздығы жайлы теорема дәлелденді. Бірмәнді шешілу шарты бұзылған жағдайда, яғни $\triangle=0$ болғанда, шеттік есептің шешуі жалғыз еместігі нақтыланды. Сонымен қоса Миттаг-Леффлер жалпыланған функцияларын Райт жалпыланған функциялары арқылы жазуды қолданып, $\lambda$ үлкен мәндерінде $\triangle$ функцияларының қасиеттері, яғни $\lambda \rightarrow \infty$ және $\lambda \rightarrow-\infty$ болғанда, оқылды. Райттың жалпыланған функциялары үшін асимптотикалық формулаларын қолданып, Штурм-Лиувилль типті шарттарымен берілген шеттік есептің меншікті мәндерінің сандарының ақырлылығы жайлы теорема анықталды.
\end{abstract}

Kiлm сөздер: бөлшек ретті дифференциалдық теңдеулер, кешікпелі аргументті дифференциалдық теңдеулер, Грин функциясы, Миттаг-Леффлер жалпыланған функциясы, Райт жалпыланған функциясы.

\section{М.Г. Мажгихова}

\section{Метод функции Грина для дифференциального уравнения дробного порядка с запаздывающим аргументом}

В статье исследована краевая задача с условиями типа Штурма-Лиувилля для линейного обыкновенного дифференциального уравнения дробного порядка с запаздывающим аргументом с постоянными коэффициентами. Условие однозначной разрешимости поставленной задачи получено в виде $\triangle \neq 0$. Для решения исследуемой задачи авторами применен метод функции Грина, в терминах которой и выписано решение краевой задачи. Функции Грина, в свою очередь, записаны в терминах обобщенной функции Миттаг-Леффлера. Доказана теорема существования и единственности решения исследуемой задачи. Отмечено, что в случае, когда условие однозначной разрешимости нарушается, то есть при $\triangle=0$, решение краевой задачи не единственно. Используя запись обобщенной функции Миттаг-Леффлера через обобщенную функцию Райта, изучены также свойства функции $\triangle$ при больших значениях $\lambda$, то есть при $\lambda \rightarrow \infty$ и $\lambda \rightarrow-\infty$. Применяя асимптотические формулы для обобщенной функции Райта, определена теорема о конечности числа собственных значений краевой задачи с условиями типа Штурма-Лиувилля.

Ключевые слова: дифференциальное уравнение дробного порядка, дифференциальное уравнение с запаздывающим аргументом, функция Грина, обобщенная функция Миттаг-Леффлера, обобщенная функция Райта. 
Green function method...

\section{References}

1 Podlubny, I. (1999). Fractional differential equations. AP, New York

2 Kilbas, A.A., Srivastava, H.M., \& Trujillo, J.J. (2006). Theory and Applications of Factional Differential Equations / Elsevier, Amsterdam.

3 Pskhu, A.V. (2005). Uravneniia $v$ chastnykh proizvodnykh drobnoho poriadka /Partial differential equations of fractional order]. Moscow: Nauka [in Russian].

4 Oldham, K.B., \& Spanier, J. (1974). The fractional calculus. N.-Y.; L.: Academiia press.

5 Miller, K.S., \& Ross, B. (1993). An introduction to the fractional calculus and fractional differential equations. John Wiley \& sons, New York.

6 Barrett, J.H. (1954). Differential equation of non-integer order. Canad. J. Math., Vol. 6, 4, 529-541.

7 Dzhrbashyan, M.M., \& Nersesyan, A.B. (1968). Drobnye proizvodnye i zadacha Koshi dlia differentsialnykh uravnenii drobnoho poriadka [Fractional derivatives and the Cauchy problem for differential equations of fractional order]. Izvestiia Akademii nauk Armianskoi SSR - News of the Academy of Sciences of the Armenian SSR, Vol. 3, 1, 3-28 [in Russian].

8 Dzhrbashyan, M.M. (1970). Kraevaia zadacha dlia differentsialnoho operatora drobnoho poriadka tipa Shturma-Liuvillia [Boundary problem for fractional differential operator of Sturm-Liouville type]. Izvestiia Akademii Nauk Armianskoi SSR - News of the Academy of Sciences of the Armenian SSR, Vol. 5, 2, 71-96 [in Russian].

9 Pskhu, A.V. (2011). Initial-value problem for a linear ordinary differential equation of noninteger order. Sbornik: Mathematics, Vol. 202, 4, 571-582.

10 Norkin, S.B. (1959). O resheniiakh lineinoho odnorodnoho differentsialnoho uravneniia vtoroho poriadka s zapazdyvaiushchim arhumentom [About the solutions of a linear homogeneous second order differential equation with delay]. Uspekhi matematicheskikh nauk - Advances in Mathematical Sciences, Vol. 14, 1, 199-206 [in Russian].

11 Bellman, R.E., Cooke, K.L. (1963). Differential-Difference Equations. New York-London: Acad. Press.

12 Elsgolts, L.E., \& Norkin, S.B. (1971). Vvedenie v teoriiu differentsialnykh uravnenii s zapazdyvaiushchim arhumentom [Introduction to the theory of differential equations with delay]. Moscow: Nauka [in Russian].

13 Myshkis, A.D. (1972). Lineinye differentsialnye uravneniia s zapazdyvaiushchim arhumentom [Linear differential equations with delay]. Moscow: Nauka [in Russian].

14 Hale, J.K., \& Lunel, S.M.V. (1993). Introduction to Functional Differential Equations. Springer, NewYork.

15 Mazhgikhova, M.G. (2018). Nachalnaia i kraevaia zadachi dlia obyknovennoho differentsialnoho uravneniia drobnoho poriadka s zapazdyvaiushchim arhumentom [Initial and boundary value problems for ordinary differential equation of fractional order with delay]. Cheliabinskii fiziko-matematicheskii zhurnal - Chelyabinsk Phisical and Mathematical Journal, Vol. 3, 1, 27-37 [in Russian].

16 Mazhgikhova, M.G. (2018). Dirichlet Problem for a Fractional-Order Ordinary Differential Equation with Retarded Argument. Differential Equations, Vol. 54, 2, 185-192.

17 Mazhgikhova, M.G. (2016). Zadacha Neimana dlia obyknovennoho differentsialnoho uravneniia drobnoho poriadka s zapazdyvaiushchim arhumentom [Neumann problem for ordinary differential equation of fractional order with delay]. Izvestiia Kabardino-Balkarskoho nauchnoho tsentra Rossiiskoi akademii nauk - The News of Kabardino-Balkarian Scientific Center of the Russian Academy of Sciences, Vol. 2, 70, 15-20 [in Russian].

18 Mazhgikhova, M.G. (2017). Zadacha Koshi dlia obyknovennoho differentsialnoho uravneniia s proizvodnoi Rimana-Liuvillia s zapazdyvaiushchim arhumentom [Cauchy problem for ordinary differential equation with Riemann-Liouville derivative with delay]. Izvestiia Kabardino-Balkarskoho nauchnoho tsentra Rossiiskoi akademii nauk - The News of Kabardino-Balkarian Scientific Center of the Russian Academy of Sciences, Vol. 1, 75, 24-28 [in Russian].

19 Mazhgikhova, M.G. (2018). Kraevye zadachi dlia lineinoho obyknovennoho differentsialnoho uravneniia drobnoho poriadka s zapazdyvaiushchim arhumentom [Boundary value problems for a linear ordinary differential equation of fractional order with delay]. sSibirskie elektronnye matematicheskie izvestiia Siberian Electronic Mathematical Reports, Vol. 15, 685-695 [in Russian]. 
20 Wiman, A. (1905). Über den Fundamentalsatz in der Teorie der Funktionen $E_{\alpha}(x)$. Acta Mathematica. Vol. 29, 1, 191-201.

21 Prabhakar, T.R. (1971). A singular integral equation with a generalized Mittag-Leffler function in the kernel. Yokohama Math. J., Vol. 19, 7-15.

22 Shukla, A.K., \& Prajapati, J.C. (2007). On a generalization of Mittag-Leffler function and its properties. J. Math. Anal. Appl. 336, 797-811.

23 Nakhushev, A.M. (2003). Drobnoe ischislenie $i$ eho primenenie [Fractional calculus and its application]. Moscow: Fizmatlit [in Russian].

24 Wright, E.M. (1935). The asymptotic expansion of the generalized hypergeometric function. J. London Math. Soc. 10, 286-293.

25 Wright, E.M. (1940). The asymptotic expansion of the generalized hypergeometric function. Proc. London Math. Soc., Vol. 46, 2, 389-408. 